\title{
Characterization and Culture of Fetal Rhesus Monkey Renal Cortical Cells
}

\author{
ALYSSA C. LEAPLEY, C. CHANG I. LEE, CYNTHIA A. BATCHELDER, MERVIN C. YODER, DOUGLAS G. MATSELL,
} AND ALICE F. TARANTAL

\begin{abstract}
Center of Excellence in Translational Human Stem Cell Research [A.C.L., C.C.I.L., C.A.B., M.C.Y., D.G.M., A.F.T.], California National Primate Research Center [A.C.L., C.C.I.L., C.A.B., A.F.T.], Departments of Pediatrics and Cell Biology and Human Anatomy [A.F.T.], University of California, Davis, California 95616; Department of Pediatrics [M.C.Y.], Indiana University School of Medicine, Indianapolis, Indiana 46202; Division of Nephrology [D.G.M.], British Columbia Children's Hospital, Vancouver, British Columbia V6H
\end{abstract} 3V4, Canada

\begin{abstract}
The renal glomerulus is composed of endothelial and mesangial cells with podocytes contributing to glomerular filtration. Podocyte damage is associated with renal disorders, thus there is interest in these cells for regenerative medicine. These studies investigated the use of extracellular matrix (ECM) to grow third trimester fetal monkey renal cortical cells and to assess mature podocytes in culture. Immunohistochemistry provided a profile of podocyte differentiation with metanephric mesenchyme and developing podocytes nestin positive and synaptopodin negative, whereas mature podocytes were positive for both markers. Primary cell cultures devoid of mature podocytes were established on plastic and renal ECM. A cell population (nestin + /synatopodin-) cultured on renal ECM showed greater proliferative potential compared with plastic with limited podocytes developing in culture over time. Further investigation of individual components of ECM (laminin, fibronectin, collagen I, or collagen IV) indicated that collagen I supported the greatest proliferation similar to renal ECM, whereas a greater number of mature podocytes (nestin+/synaptopodin + ) were observed on fibronectin. These results suggest that (1) culture of fetal monkey podocytes can be accomplished, (2) renal ECM and collagen I can support renal cortical cells in vitro, which may recapitulate the developing kidney in vivo, and (3) fibronectin can support podocyte differentiation in vitro. (Pediatr Res 66: 448-454, 2009)
\end{abstract}

$\mathrm{P}$ odocytes are specialized terminally differentiated cells that interact with the glomerular capillaries, neighboring podocytes, and the glomerular basement membrane by interdigitating foot processes and slit diaphragms (1-3). Podocytes are typically fully differentiated in newborns, while undifferentiated podocytes are found in fetuses with the exception of some fish and shark species that retain the capability for growth of new glomeruli with maturation and aging (4). These cells differentiate from the metanephric mesenchyme and pass through four stages of glomerular development until they form the final mature phenotype, which can no longer divide $(2,5)$. Podocytes are required for maintenance and integrity of the glomerular filtration barrier. They are commonly damaged or

Received March 18, 2009; accepted June 14, 2009.

Correspondence: Alice F. Tarantal, Ph.D., California National Primate Research Center, Pedrick and Hutchison Roads, University of California, Davis, CA; e-mail: aftarantal@primate.ucdavis.edu

Supported by NIH Grants HL069748 (Center of Excellence) and RR00169 (Primate Center base operating grant). destroyed in renal disease such as congenital nephritic syndrome in humans and are an actively studied cell type (6).

Urinary tract obstruction in fetal rhesus monkeys during active nephrogenesis has been shown to result in tubular cystic transformation and irregular collecting duct epithelialmesenchymal transition, as seen in human urinary tract obstruction and renal dysplasia $(7,8)$. Notably, obstructed kidneys also showed a severe disruption of normal glomerular development with consequent podocyte apoptosis and loss (9). This in utero monkey model of human obstructive renal dysplasia is particularly important because monkeys exhibit active nephrogenesis comparable to humans during similar developmental time periods, with a well-demarcated nephrogenic zone and identifiable metanephric mesenchyme, ureteric bud, S-shaped nephrons, and maturing glomeruli $(10,11)$. Thus, the fetal rhesus monkey model can play an essential role in understanding the role of podocytes in the prenatal pathogenesis of obstructive renal dysplasia and to develop potential cell-based therapies using podocyte progenitors for human applications. However, culture conditions to obtain fetal rhesus monkey podocyte progenitors have not yet been established, nor is there a differentiation assay to test the ability of the cultured cells to differentiate into mature podocytes once they have been transplanted into a kidney compromised by disease.

In this study, immunohistochemical analysis of third trimester fetal monkey kidneys provided a phenotypic profile of podocyte maturation. This developmental pattern was then used to analyze and identify cultured third trimester fetal monkey renal cortical cells. Finally, the effects of renal extracellular matrix (ECM) and its individual components (laminin, fibronectin, collagen I, and collagen IV) were investigated for their potential to support the growth and differentiation of cultured renal cortical cells.

\section{METHODS}

Animals. All animal procedures conformed to the requirements of the Animal Welfare Act, and protocols were approved before implementation by the Institutional Animal Care and Use Committee at the University of California, Davis. Normally cycling, adult female rhesus monkeys (Macaca 
mulatta $)(n=3)$ with a history of prior pregnancy were bred and identified as pregnant, using established methods (12). Pregnancy in the rhesus monkey is divided into trimesters by 55-d increments with term delivery at $165 \pm 10 \mathrm{~d}$ (13). Activities related to animal care (diet, housing) were performed as per standard California National Primate Research Center (CNPRC) operating procedures. Normal fetal growth and development was confirmed by ultrasound (14), and fetuses were delivered by hysterotomy for tissue harvests in the early third trimester ( $120 \mathrm{~d}$ gestation) as previously described (7).

Immunohistochemistry. Fetal kidneys were collected, and one half of each kidney was placed in optimum cutting temperature (OCT) embedding compound (Sakura, Japan) and quick frozen over liquid nitrogen. Sections $(4 \mu \mathrm{m})$ from OCT-embedded kidneys were used for the analyses. Slides were placed in PBS for 5 min, then fixed in cold methanol (Fisher Chemical, Fairlawn, NJ) for $10 \mathrm{~min}$, and allowed to air dry before blocking with $2 \%$ serum of the appropriate secondary antibody (incubated for $20 \mathrm{~min}$ at room temperature). Primary and secondary antibodies were diluted in PBS with 1\% BSA (Invitrogen, Carlsbad, CA). Excess blocking buffer was removed and sections incubated with primary antibodies overnight at $\leq 4^{\circ} \mathrm{C}$ in a moisture chamber. The following primary antibodies and dilutions were used: rabbit anti-nestin (1:500; Chemicon, Temecula, CA); goat anti-synaptopodin (1:100; Santa Cruz Biotech, Santa Cruz, CA); mouse anti-CD31 (1:100; Dako, Carpinteria, CA); mouse anti-synaptopodin (1:100; Progen, Heidelberg, Germany); rabbit anti-nephrin (1:500; Proscience, San Diego, CA); mouse anti-podocalyxin (1:500; Invitrogen); mouse anti-WT-1 (predilute; Invitrogen); mouse antivimentin (1:500; BD Biosciences, Palo Alto, CA); and goat anti-podocin (1:100; Santa Cruz Biotech). Excess primary antibody solution was removed before the secondary antibody was added and incubated in the dark at room temperature for $1 \mathrm{~h}$. Secondary antibodies included the following: Alexa Fluor goat-rabbit 488 and 594, donkey-goat 594, and goat-mouse 488 and 594 (1:500; Invitrogen). Double staining was accomplished by the application of a mixture of primary antibodies overnight at $\leq 4^{\circ} \mathrm{C}$, followed by a mixture of the appropriate secondary antibodies for $1 \mathrm{~h}$ in the dark at room temperature. Antibody dilutions for double staining were the same as described above for single-stain preparations. Coverslips were then mounted with Prolong Gold mounting medium with the nuclear stain 4'-6-diamidino-2-phenylindole (DAPI) (Invitrogen). All photographic images were obtained using an Olympus Bx61 Microscope (Olympus America Inc, Center Valley, PA) with a CoolSNAP HQ black and white camera.

Cell culture. One half of each kidney was separated into cortex and medulla at the corticomedullary border with the cortical components placed in DB20 medium composed of DMEM (Invitrogen) with 20\% fetal bovine serum (FBS), $1 \%$ penicillin-streptomycin, and 1\% L-glutamine (all from Invitrogen). The tissue was ground using the frosted end of sterile glass slides, cultured in DB20 until 70\% confluent, and subsequently passaged using $0.05 \%$ trypsin-EDTA (Invitrogen). Primary culture cells were also cryopreserved for further study using a standardized controlled rate cryopreservation protocol (CryoMed, Thermo Scientific) in FBS and 10\% DMSO (SigmaAldrich Chemical Co., St. Louis, MO).

To identify the culture conditions supporting podocyte progenitor growth, cryopreserved cells from the primary cultures (P0) were plated on various substrates including plastic, laminin, fibronectin, collagen I, and collagen IV (BD Biosciences). Renal ECM was obtained as follows: cultured cortical cells were grown in DB20 on collagen I until 4-5 d postconfluence, washed once with PBS, incubated with $0.1 \mathrm{~N}$ ammonia solution in water (Fisher Chemicals) for $10 \mathrm{~min}(15,16)$, and washed with PBS three times before plating the cells.

Cortical cells from three animals (primary cultures) were plated individually onto the six substrates in six-well plates at $1 \times 10^{3}$ cells $/ \mathrm{cm}^{2}$ and in duplicate. Cells were passaged when $\sim 70 \%$ confluence was achieved and through five passages. At each passage, cells were counted in duplicate on a hemacytometer and an aliquot collected for quantitative RT-PCR analysis (see below). Cells at each passage were also plated on two-well chamber slides (Nunc Lab-Tek, Fisher) coated with each of the experimental substrates, grown until $\sim 70 \%$ confluent, fixed with ice-cold methanol, and stored at $\leq-20^{\circ} \mathrm{C}$ until immunocytochemical analysis.

Immunocytochemistry. The primary and secondary antibodies identified above for immunohistochemistry were used for analysis of cultured cells on the different substrates. Primary antibodies were diluted as described earlier, applied to chamber slides, and incubated for $1 \mathrm{~h}$ at room temperature. The primary antibody was removed and the slide washed twice with PBS before application of the appropriate secondary antibody (1:200) for $1 \mathrm{~h}$ in the dark at room temperature. After removal of the secondary antibody, the slide was washed once with PBS and a coverslip was placed with Prolong Gold with DAPI for nuclear staining. For double staining, the primary antibodies were applied together in the appropriate dilutions and incubated for $1 \mathrm{~h}$ at room temperature. After removal of the primary antibody, slides were washed twice with PBS, and the Alexa-Fluor 594 antibody of the appropriate host (1:200)
Table 1. PCR primers for assessment of renal podocyte progenitors

\begin{tabular}{lll}
\hline \multicolumn{1}{c}{ Gene } & Primer & \multicolumn{1}{c}{ Sequence } \\
\hline HPRT1 & Forward & 5'-TTTTATCAGACTGAAGAGCTATTGTAATGA-3' \\
& Reverse & 5'-CATCGTTTTGCAGTGTCAATTAT-3' \\
\multirow{2}{*}{ Nephrin } & Forward & 5'-TGGGAGAGAGACTGGGAGAAGA-3' \\
& Reverse & 5'-CAGAACTGGTGCTGTCTCCA-3' \\
\multirow{2}{*}{ Nestin } & Forward & 5'-TGGCAAGAGGCCGGTACA-3' \\
& Reverse & 5'-CCGTATTTGTCCTTCACCTTC-3' \\
\multirow{2}{*}{ Podocalyxin } & Forward & 5'-AAGGACCAGCAGCGGCTAA-3' \\
& Reverse & 5'-CATCACTTCCAGTGTTGGGTTGT-3' \\
\multirow{2}{*}{ Podocin } & Forward & 5'-GCAGCTCTGAGGATGGAGAG-3' \\
& Reverse & 5'-GGACCTCATCCACGTCCAC-3' \\
\multirow{2}{*}{ Synaptopodin } & Forward & 5'-CAGATTGGGCCAGAGCACTAG-3' \\
& Reverse & 5'-TTGGACGCCACGGGAAT-3' \\
\multirow{2}{*}{ WT-1 } & Forward & 5'-CTTCAGAGGCATTCAGGATGTG-3' \\
& Reverse & 5'-TCTCAGATGCCGACCGTACA-3' \\
\hline
\end{tabular}

HPRT1, hypoxanthine phosphoribosyltransferase 1 (housekeeping gene).

was added and incubated in the dark for $1 \mathrm{~h}$. The first secondary antibody was removed, the slide washed twice with PBS, and stained with Alexa-Fluor 488 antibody of the appropriate host (1:200) for $1 \mathrm{~h}$ in the dark at room temperature, washed once with PBS, and a coverslip placed.

Nestin and synaptopodin gene expression was examined under fluorescent microscopy. Where possible, images were collected ( $>20$ per culture slide) for a semiquantitative analysis of protein expression. Total cells per field were counted based on DAPI nuclear staining and nestin positive cells identified based on presence of appropriate fluorescence signal. The percentage of positive cells was calculated and applied to a 0 to 4 scoring system as follows: -, no evidence of expression; + , weak expression, $<25 \%$ positive cells; ++ modest expression, $25-49 \%$ positive; +++ , moderate expression, $50-74 \%$ positive; and ++++ , strong levels of expression, $\geq 75 \%$ positive.

Quantitative PCR. Cell pellets collected from the above cultures at each of five passages were processed for total RNA using a Qiagen RNeasy Mini Kit according to the manufacturer's recommendations (Qiagen, Valencia, CA). The cDNA was processed using a Qiagen Sensiscript RT kit and the protocol according to the manufacturer's recommendations. For quantitative PCR analysis, primers were designed for each target gene (see Table 1). Real-Time quantitative PCR analysis was carried out in 96-well optical plates using the 7900 ABI Sequence Detection System (Applied Biosystems, Foster City, CA) and the QuantiTect SYBR Green PCR Kit (Qiagen) according to the manufacturer's protocols. Primers for each target gene were designed to span an intron and thus eliminate the possibility of amplifying genomic DNA templates remaining in the RNA preparations (Table 1). PCR reactions were run in duplicate in separate wells and contained $1 \times$ SYBR Green master mix and $500 \mathrm{nM}$ of forward and reverse primers in a $25-\mu \mathrm{L}$ reaction volume. The PCR protocol consisted of one cycle of $2 \mathrm{~min}$ at $50^{\circ} \mathrm{C}, 15 \mathrm{~min}$ at $95^{\circ} \mathrm{C}$, followed by 40 cycles at $15 \mathrm{~s}$ at $95^{\circ} \mathrm{C}$, and $60 \mathrm{~s}$ at $60^{\circ} \mathrm{C}$. RNA expression was quantified relative to the housekeeping gene, HPRT1, and to normalize the amount of sample RNA and to compensate for any potential sample variation. Positive and negative controls (water) were included in each run. Differences in gene expression were determined as described in the User's Bulletin \#2 (P/N 4303859) (Applied Biosystems, updated 2001).

Data analysis. Data are shown as mean \pm SEM. Significance was determined by $t$ test analysis at $p \leq 0.05$.

\section{RESULTS}

Rhesus monkey fetuses were delivered by hysterotomy for tissue harvest in the early third trimester ( $120 \mathrm{~d}$ gestation), and specimens collected and processed for immunohistochemistry and cell culture.

Immunohistochemistry. The nephrogenic zone of the fetal kidney includes metanephric mesenchyme, branching ureteric buds, and the four stages of the developing glomeruli (vesicle, S-shape, comma, and mature) $(17,18)$ (Fig. 1A). Immunohistochemical analyses showed metanephric mesenchyme in the nephrogenic zone expressed nestin, WT-1, and vimentin, whereas developing podocytes at the S-shape and comma phase of glomerular development expressed nestin, WT-1, 

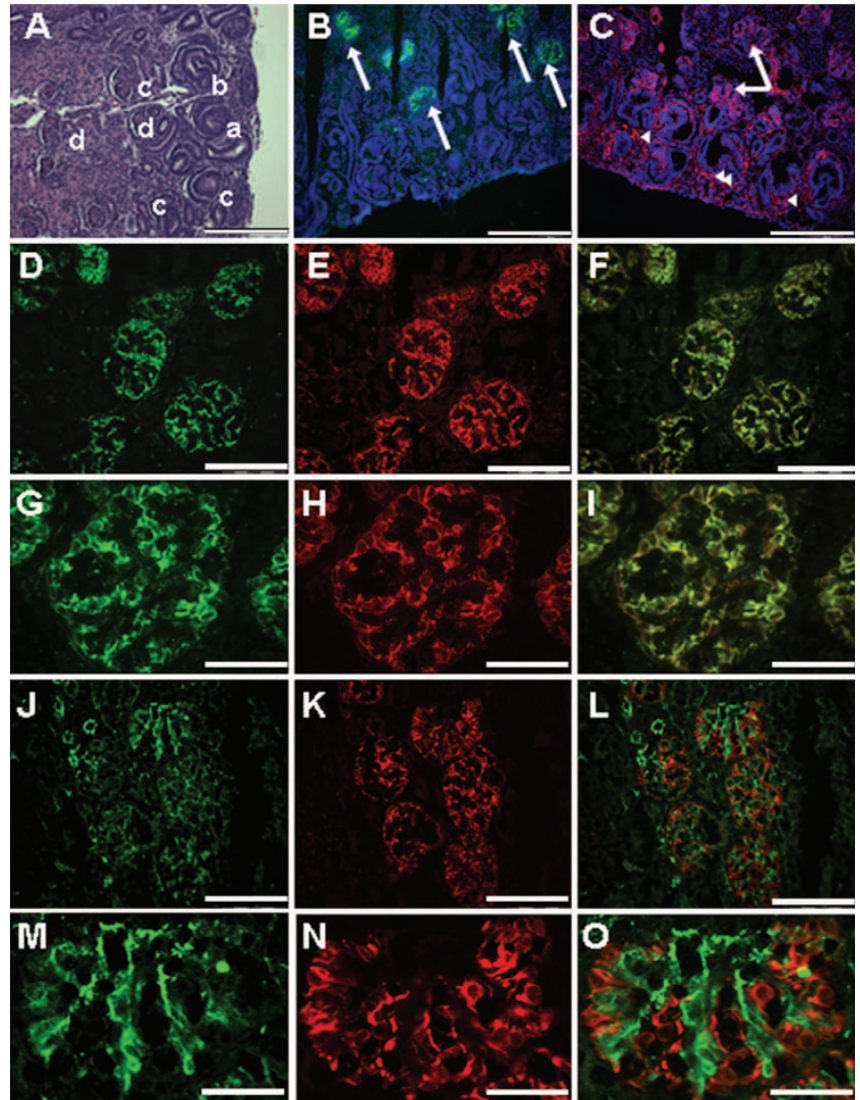

Figure 1. Renal morphology and immunohistochemistry-early third trimester fetal monkey kidney and nephrogenic zone. (A) The fetal monkey kidney in the early third trimester shows a clear nephrogenic zone in the cortical region with the ureteric bud (arrowhead) and the metenephric mesenchyme (arrow) evident. The four stages of developing glomeruli are also present and represent the vesicle (a), S-shape (b), capillary (c), and mature (d) glomerulus. Hematoxylin and eosin (H\&E). $(B, C)$ The nephrogenic zone is shown with the nuclei stained with DAPI (blue), metanephric mesenchyme (arrowheads), developing podocytes, and mature podocytes (arrows) positive for synaptopodin (green) and nestin (red). (D-I) Mature podocytes in terminally differentiated glomeruli are positive for nestin (red) and synaptopodin (green). $(\mathrm{J}-\mathrm{O})$ The endothelial marker CD31 (green) is not evident with the nestin positive cells $($ red $)$ in these specimens $(A-C, \times 20$, bar $=100 \mu \mathrm{m} ; D-F, \times 40$, bar $=$ $50 \mu \mathrm{m} ; G-I, \times 100$, bar $=20 \mu \mathrm{m} ; J-L, \times 40$, bar $=50 \mu \mathrm{m} ; M-O, \times 100$, bar $=20 \mu \mathrm{m})$.

vimentin, nephrin, and podocalyxin. Mature podocytes at the final phase of glomerular development expressed nestin, WT-1, vimentin, nephrin, podocalyxin, synaptopodin, and podocin similar to findings in humans $(5,19-23)$. Because podocytes function in close proximity to endothelial cells and express many of the same markers, CD31, which is expressed on endothelial cells (5), was used to distinguish between the two cell types. Using double-staining techniques, cells positive for nestin and synaptopodin were shown to be negative for CD31 (Fig. 1). These findings provided a profile of podocyte differentiation that was then used to assess renal cortical cells grown in vitro.

Effects of renal ECM. Cells were plated at a cell density of $1 \times 10^{3}$ cells $/ \mathrm{cm}^{2}$ and grown on plastic and ECM prepared by decellularizing renal cortical cells. Cell morphology when grown on plastic or renal ECM (passages 1-3) was similar to published descriptions of podocyte progenitors in mouse and

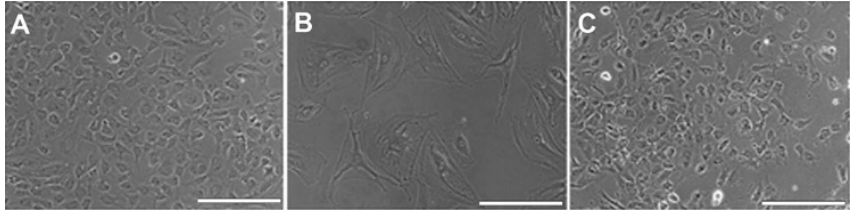

Figure 2. Morphology of cultured renal cortical cells. (A) Through passage 4 , all substrates studied showed cells with the "cobblestone" appearance suggestive of undifferentiated podocytes. $(B)$ At passage 5, cultures contained cells that had a differentiated podocyte cell morphology with arborization, processes extended from cell bodies, and a large cytoplasmic to nuclear volume ratio. $(C)$ Renal ECM contained cells with an undifferentiated podocyte morphology through all passages $(1$ through 5$)(\times 20$, bar $=100 \mu \mathrm{m})$.

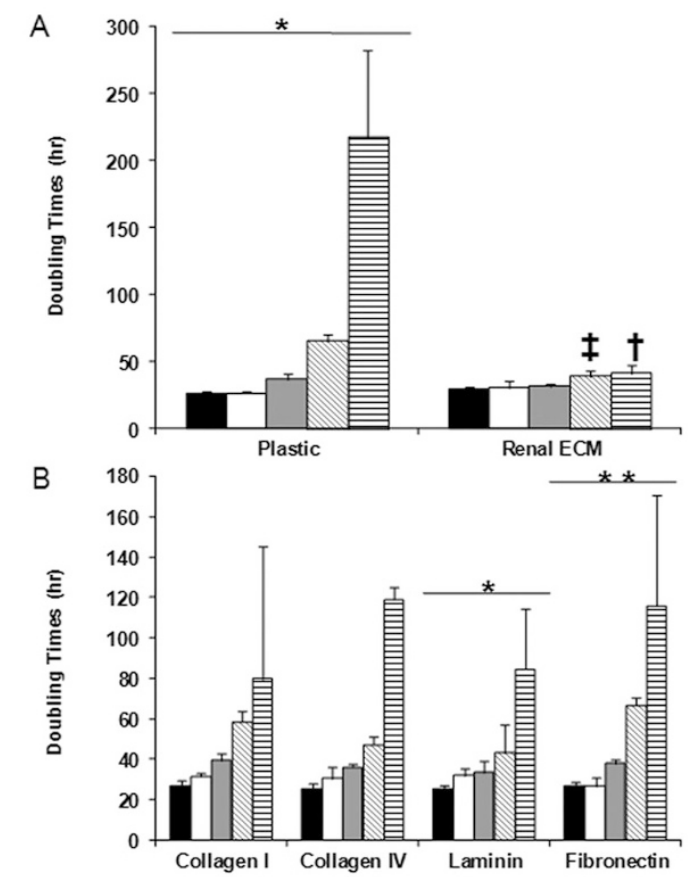

Figure 3. Population doubling times-renal cortical cells on renal ECM. (A, B) (black, P1; white, P2; gray, P3; diagonal hatched, P4; vertical hatched, P5). The $Y$-axis represents population doubling times (h). The population doubling times suggest that in the early passages, the substrate did not alter the growth capabilities of the cells. For passages 4 and 5, renal ECM was shown to support the optimal population doubling times. Comparisons were made of passage 1 (P1) vs passage 5 (P5) for each substrate; ${ }^{*} p<0.05$, ** $p<0.01$. Comparisons of renal ECM with plastic are shown at passage $4\left({ }^{\ddagger} p<0.01\right)$, and renal ECM with plastic, fibronectin, and laminin at passage $5\left({ }^{\dagger} p<0.05\right)$.

human podocyte cultures (19) with the classic "cobblestone" appearance and a low cytoplasmic to nuclear volume ratio (Fig. 2). During passages 4-5, cells plated on plastic showed a more differentiated morphology characterized by arborization, processes extending from the cell body, and a high cytoplasmic to nuclear volume ratio (Fig. 2). Cells with a differentiated morphology showed limited proliferation and an extended population doubling time (80-200 h; passage 5). However, renal ECM consistently supported proliferation of cells retaining the "cobblestone" morphology at a significantly higher growth rate compared with those plated on plastic $(p<$ 0.05 ) (Fig. 3). The population doubling time was maintained with renal ECM at passages 4 and 5 with average doubling times of $39.4 \pm 4.2 \mathrm{~h}$ and $41.6 \pm 6.4 \mathrm{~h}$, respectively. 


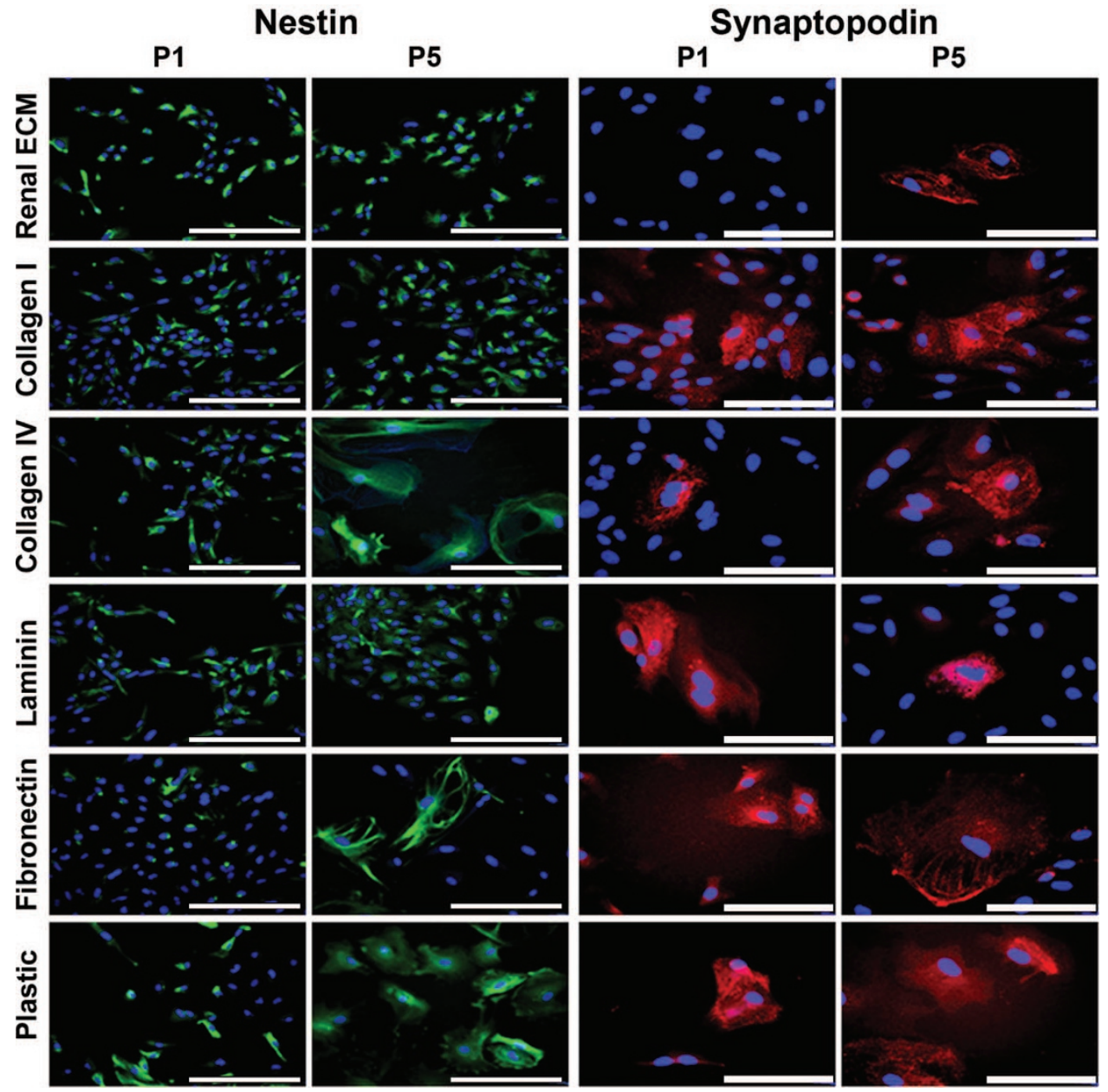

Figure 4. Immunocytochemistry-cultured cortical cells. Cells from passage 1 (P1) grown on each of the substrates included nestin positive cells (green) $(\times 20$, bar $=100 \mu \mathrm{m})$. Nuclei stained with DAPI (blue). At passage 5 (P5), nestin positive cells decreased on all substrates except renal ECM and collagen I. Cells from P1 cultured on five of six substrates contained very few cells that were synaptopodin positive $(\mathrm{red}, \times 40$, bar $=50 \mu \mathrm{m})$. None of the cells grown on renal ECM showed evidence of synaptopodin expression. At P5, there was an increase in synaptopodin positive cells suggesting a more mature podocyte phenotype.
Table 2. Nestin and synaptopodin expression in renal progenitor cells at passages 1 and 5

\begin{tabular}{ccccccc}
\hline & \multicolumn{2}{c}{ Nestin } & & \multicolumn{2}{c}{ Synaptopodin } \\
\cline { 2 - 3 } \cline { 5 - 6 } Extracellular matrix & Passage 1 & Passage 5 & & Passage 1 & Passage 5 \\
\hline Collagen I & +++ & ++ & & + & ++ \\
Collagen IV & +++ & + & & + & ++ \\
Fibronectin & ++++ & ++ & & + & ++++ \\
Laminin & ++++ & ++ & & + & ++ \\
Plastic & ++++ & + & & + & ++ \\
Renal ECM & ++++ & +++ & & - & + \\
\hline
\end{tabular}

++++ , strong $;++$, moderate; ++ , modest; + , weak;,- no evidence.

At passage 1 , a majority of cells $(\sim 75 \pm 10 \%)$ were nestin positive when grown on plastic or renal ECM with no significant differences between the groups noted (Fig. 4). Nestin expression for cells plated on plastic diminished with advancing passage (Table 2). However, cells plated on renal ECM maintained nestin expression over 5 passages. In contrast, cells plated on renal ECM did not show synaptopodin expression at passage 1 , and very few synaptopodin positive cells were observed in culture at passage 5. A slight increase in synaptopodin expression for cells plated on plastic was observed over 5 passages. Taken together, renal ECM was shown to support proliferation of nestin positive renal cortical cells without increasing synaptopodin expression.

Effects of ECM components. Cells were cultured in individual ECM components (collagen I, collagen IV, fibronectin, laminin) to more specifically characterize findings. Cells cultured on collagen IV and laminin showed growth rates comparable with renal ECM over 4 passages, followed by a significant increase in doubling times at passage $5(p<0.05)$ (Fig. 3). A significant increase in the population doubling times of renal cells cultured on fibronectin $(115.6 \pm 22.7 \mathrm{~h})$ was also observed at passage 5 when compared with renal ECM (41.0 $\pm 6.4 \mathrm{~h}, p<0.05)$ (Fig. 4). Similar to plastic, cells cultured on all ECM substrates assessed showed a high quantity of cells suggestive of mature podocyte morphology as noted above for cells grown on plastic.

At passage 1, a majority of cells $(\sim 75 \pm 10 \%)$ were nestin positive when grown on each of the four substrates studied with no significant differences between the groups noted (Fig. 4 and Table 2), with a similar decline with advancing passage. Conversely, very few cells on any of the substrates assessed were synaptopodin positive at passage 1 . With advancing passage, an increase in the quantity of synaptopodin positive cells was noted, particularly when grown on fibronectin (Figs. 4 and 5 and Table 2). These synaptopodin positive cells were also positive for nestin and WT-1 and displayed a differentiated podocyte morphology. These observations suggest that fibronectin supports differentiation of renal cortical cells toward a podocyte lineage.

Quantitative PCR. All cells grown on the different substrates (passages 1 through 5) were positive for podocin, 

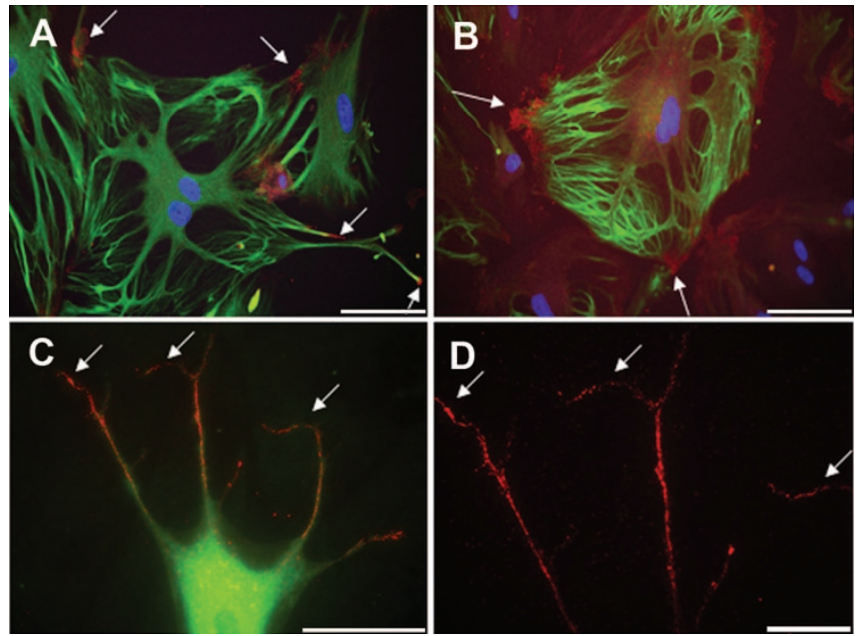

Figure 5. Immunocytochemistry-cultured cortical cells on fibronectin and renal ECM. Cells from passage 5 cultured on fibronectin contained more synaptopodin positive cells (red) (arrow) than those cultured on other substrates. Nuclei stained with DAPI (blue) $(A-D)$. Synaptopodin positive cells were positive for nestin (green) and showed morphology consistent with mature podocytes $(A-C)$. $(A-B, \times 20$, bar $=100 \mu \mathrm{m} ; C, \times 60$, bar $=50 \mu \mathrm{m}$; $D, \times 100$, bar $=20 \mu \mathrm{m})$.

podocalyxin, nestin, nephrin, synaptopodin, and WT-1 when evaluated by quantitative PCR (see Table 1). Synaptopodin expression increased on all substrates with advancing passage and was particularly increased on plastic $(p<0.01)$ and fibronectin $(p<0.05)$ suggesting maturation over time toward a mature podocyte phenotype (Fig. 6). Nestin expression was noted to decline when cells were grown on plastic or laminin $(p<0.05)$ suggesting a loss of proliferative potential typically associated with podocyte progenitor differentiation.

\section{DISCUSSION}

Early third trimester fetal monkey renal cortical cells were cultured on six different substrates including plastic, laminin, fibronectin, collagen I, collagen IV, and renal ECM through five passages. Results of these studies suggest that (1) these cells can be grown and maintained in culture, (2) renal ECM and collagen I may best support a podocyte progenitor population in vitro, which can recapitulate the developing kidney in vivo, and (3) fibronectin may best support podocyte differentiation in vitro. These cells could prove useful for kidney regeneration protocols and in translational studies with nonhuman primate models where a loss of podocytes has been shown to occur, such as fetal obstructive renal disease $(7,9,24)$.

The metanephric mesenchyme of the developing kidney contains progenitors that differentiate into mature podocytes, mesangial cells, and tubular epithelium. It is currently unknown whether endothelial cells are recruited into the kidney by early podocyte signaling or whether these progenitors are present locally (25). Similarly, it is unclear whether there is a specific progenitor population that differentiates toward a mesangial lineage or whether these cells differentiate from an endothelial progenitor or from a single progenitor with the potential to form mesangial cells and podocytes $(1,26)$. Several studies have been performed to assess the molecular features of podocytes in vivo, but culture conditions have not
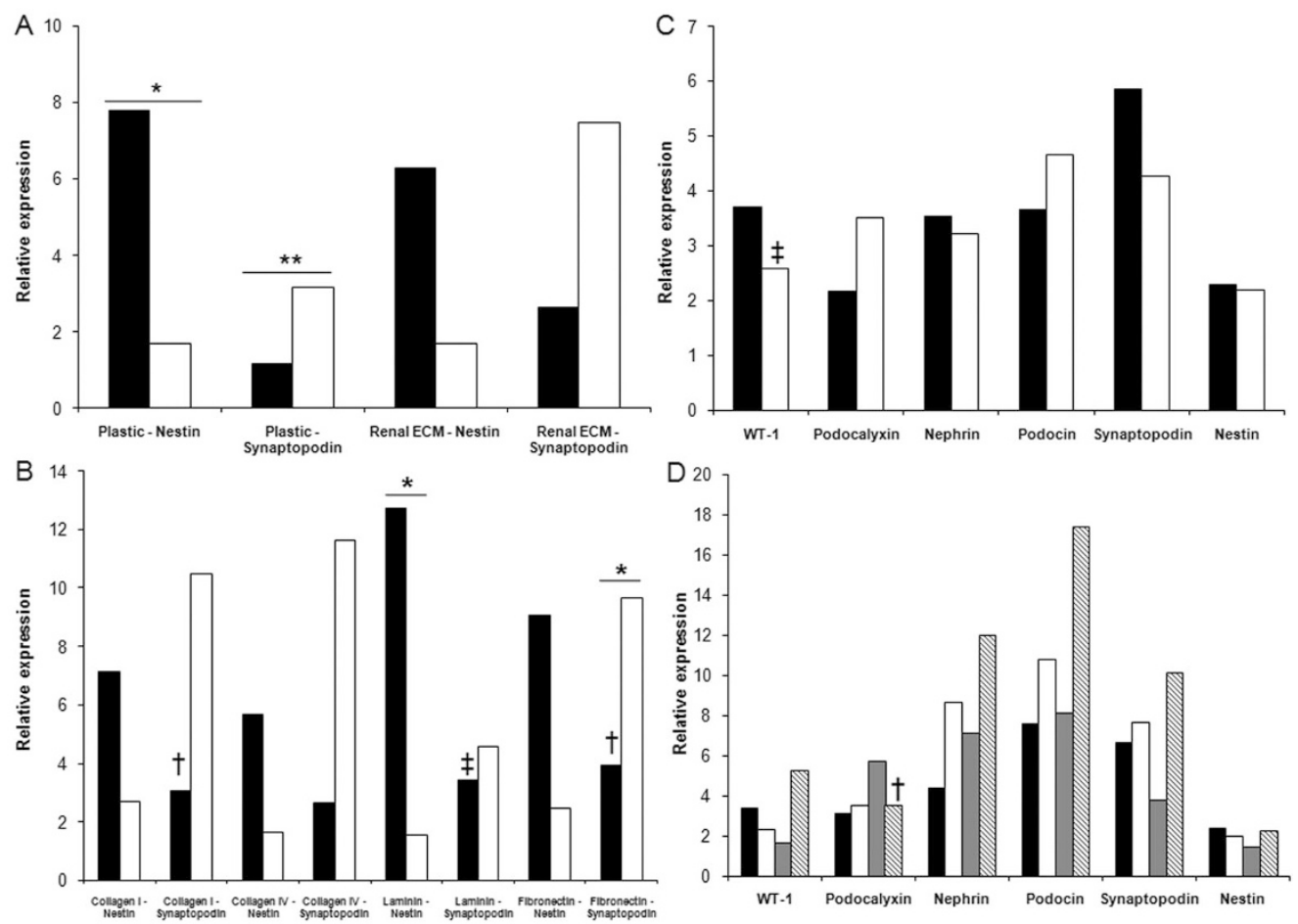

Figure 6. Quantitative PCR—synaptopodin and nestin. Although synaptopodin expression increased $(A)$, nestin expression decreased $(B)$ in cells grown on all substrates studied (black column, P1; white column, P5). Furthermore, podocyte specific expression was present in cells at P5 (C) (black, plastic; white, renal $\mathrm{ECM})(D)$ (black, collagen I; white, collagen IV; gray, laminin; hatched, fibronectin). Passage 1 (P1) compared with passage 5 (P5) $(* p<0.05$, ** $p<0.01$ ). Comparisons of plastic to other substrates $\left({ }^{\dagger} p<0.05,{ }^{\ddagger} p<0.01\right)$. 
yet been established that allow the maintenance of these cells in vitro. In this study, we used established human podocyte markers including nestin for podocyte progenitors and synaptopodin for mature podocytes. Nestin is an intermediate filament protein that was first discovered in neural progenitor cells (27), then found on mature podocytes in the rat (20), mouse (28), and human $(5,29)$ and currently shown to be expressed in the metanephric mesenchyme of the developing fetal monkey kidney. Conversely, synaptopodin has only been observed in mature, fully differentiated podocytes in fetuses and adults from several mammalian species $(19,21)$. By using these two markers, we were able to determine the developmental status of renal cortical cells from the nephrogenic zone when grown in culture and anticipate that these markers will be useful for determination of cell fate in preclinical cellbased studies focused on fetal kidney repair.

Podocytes and endothelial cells produce components of the glomerular basement membrane on which these cells mature and function. Therefore, by using a decellularized confluent cortical cell culture method on which to grow podocytes, the ECM allowed proliferation and maintenance of progenitors in vitro. However, renal ECM is challenging to grow with a high degree of consistency and is much more delicate than the commercial substrates currently available. The average population doubling times over the five passages studied showed that renal ECM can maintain a higher quantity of proliferative cells when compared with the other substrates. Furthermore, renal ECM maintained podocyte progenitor morphology over a longer period of time and with a high quantity of nestin positive and fewer synaptopodin positive cells. Collagen I, the most commonly used substrate for podocyte culture $(30,31)$, was shown to be the next best substrate for cell growth and proliferation and provided consistent population doubling times, with later passages containing cells with a podocyte progenitor morphology strongly positive for nestin. Conversely, the other commercial substrates were more effective for driving podocyte progenitors toward a mature, terminally differentiated state. Nestin and synaptopodin staining support that the cells were an immature population in the early passages and then matured with subsequent passages. The cultured cortical cells were also shown to be positive for podocyte markers when evaluated by quantitative PCR and displayed the characteristic features of podocyte progenitors with the ability to differentiate into mature podocytes (19) (see Figs. 4-6).

During the past few decades, it has been shown that a large number of renal disorders result in glomerular pathologies and most appear to be specific to the podocyte (32-37). Podocyte loss by necrosis, apoptosis, or detachment results in glomerulosclerosis and eventually leads to end stage renal disease (37). Further, obstructive uropathy, which is one of the more common renal disorders in infants and children, results in multiple cysts and an associated podocyte loss (9). There has been some debate as to whether injured podocytes can be replaced (38), and currently, there is no definitive technique available to replace podocytes damaged by disease $(32,33)$.

The results of this study suggest that renal ECM and collagen I are effective cell culture substrates for maintaining podocyte progenitors in vitro, with fibronectin serving as a culture substrate, which can provide more mature, terminally differentiated cells. For tissue regeneration purposes, podocyte progenitors may prove useful to repair damaged kidneys. The fetal rhesus monkey model of obstructive renal disease $(7,9)$ provides a unique preclinical model to develop and test such strategies, and it may provide new insights for kidney regeneration and repair that can be translated to the human clinical setting.

\section{REFERENCES}

1. Quaggin SE, Kreidberg JA 2008 Development of the renal glomerulus: good neighbors and good fences. Development 135:609-620

2. Saxen L 1987 Organogenesis of the Kidney. Cambridge University Press, Cambridge, pp 135-142

3. Miner JH 1999 Renal basement membrane components. Kidney Int 56:2016-2024

4. Haller H, de Groot K, Bahlmann F, Elger M, Fliser D 2005 Stem cells and progenitor cells in renal disease. Kidney Int 68:1932-1936

5. Bertelli E, Regoli M, Fonzi L, Occhini R, Mannucci S, Ermini L, Toti P 2007 Nestin expression in adult and developing human kidney. J Histochem Cytochem 55:411421

6. Krtil J, Platenik J, Kazderova M, Tesar V, Zima T 2007 Culture methods of glomerular podocytes. Kidney Blood Press Res 30:162-174

7. Tarantal AF, Han VK, Cochrum KC, Mok A, daSilva M, Matsell DG 2001 Fetal rhesus monkey model of obstructive renal dysplasia. Kidney Int 59:446-456

8. Butt MJ, Tarantal AF, Jimenez DF, Matsell DG 2007 Collecting duct epithelialmesenchymal transition in fetal urinary tract obstruction. Kidney Int 72:936-944

9. Matsell DG, Mok A, Tarantal AF 2002 Altered primate glomerular development due to in utero urinary tract obstruction. Kidney Int 61:1263-1269

10. Lee CI, Goldstein O, Han VK, Tarantal AF 2001 IGF-II and IGF binding protein (IGFBP-1, IGFBP-3) gene expression in fetal rhesus monkey tissues during the second and third trimesters. Pediatr Res 49:379-387

11. Rodriguez CG, Makori N, Cukierski MA, Hendrickx AG 1996 Development of the definitive kidney in the cynomolgus monkey (Macaca fascicularis). J Med Primatol 25:122-132

12. Tarantal AF, Hendrickx AG 1988 Use of ultrasound for early pregnancy detection in the rhesus and cynomolgus macaque (Macaca mulatta and Macaca fascicularis). J Med Primatol 17:105-112

13. Tarantal AF, Gargosky SE 1995 Characterization of the insulin-like growth factor (IGF) axis in the serum of maternal and fetal macaques (Macaca mulatta and Macaca fascicularis). Growth Regul 5:190-198

14. Tarantal AF 2005 Ultrasound imaging in rhesus (Macaca mulatta) and long-tailed (Macaca fascicularis) macaques: reproductive and research applications. In: WolfeCoote S (ed) The Laboratory Primate. Elsevier Academic Press, Boston, pp 317-351

15. Gospodarowicz D, Delgado D, Vlodavsky I 1980 Permissive effect of the extracellular matrix on cell proliferation in vitro. Proc Natl Acad Sci USA 77:4094-4098

16. Matsubara T, Tsutsumi S, Pan H, Hiraoka H, Oda R, Nishimura M, Kawaguchi H, Nakamura K, Kato Y 2004 A new technique to expand human mesenchymal stem cells using basement membrane extracellular matrix. Biochem Biophys Res Commun 313:503-508

17. Burrow CR 2000 Regulatory molecules in kidney development. Pediatr Nephrol 14:240-253

18. Horster MF, Braun GS, Huber SM 1999 Embryonic renal epithelia: induction, nephrogenesis, and cell differentiation. Physiol Rev 79:1157-1191

19. Shankland SJ, Pippin JW, Reiser J, Mundel P 2007 Podocytes in culture: past, present, and future. Kidney Int 72:26-36

20. Zou J, Yaoita E, Watanabe Y, Yoshida Y, Nameta M, Li H, Qu Z, Yamamoto T 2006 Upregulation of nestin, vimentin, and desmin in rat podocytes in response to injury. Virchows Arch 448:485-492

21. Mundel P, Gilbert P, Kriz W 1991 Podocytes in glomerulus of rat kidney express a characteristic 44 KD protein. J Histochem Cytochem 39:1047-1056

22. Pavenstadt H, Kriz W, Kretzler M 2003 Cell biology of the glomerular podocyte. Physiol Rev 83:253-307

23. Roselli S, Gribouval O, Boute N, Sich M, Benessy F, Attie T, Gubler MC, Antignac C 2002 Podocin localizes in the kidney to the slit diaphragm area. Am J Pathol 160:131-139

24. Chevalier RL, Thornhill BA, Wolstenholme JT, Kim A 1999 Unilateral ureteral obstruction in early development alters renal growth: dependence on the duration of obstruction. J Urol 161:309-313

25. Nishinakamura R 2008 Stem cells in the embryonic kidney. Kidney Int 73:913-917

26. Takeda S, Rogers SA, Hammerman MR 2006 Differential origin for endothelial and mesangial cells after transplantation of pig fetal renal primordia into rats. Transpl Immunol 15:211-215

27. Lendahl U, Zimmerman LB, McKay RD 1990 CNS stem cells express a new class of intermediate filament protein. Cell 60:585-595

28. Chen J, Boyle S, Zhao M, Su W, Takahashi K, Davis L, Decaestecker M, Takahashi T, Breyer MD, Hao CM 2006 Differential expression of the intermediate filament protein nestin during renal development and its localization in adult podocytes. J Am Soc Nephrol 17:1283-1291 
29. Perry J, Ho M, Viero S, Zheng K, Jacobs R, Thorner PS 2007 The intermediate filament nestin is highly expressed in normal human podocytes and podocytes in glomerular disease. Pediatr Dev Pathol 10:369-382

30. Yaoita E, Kurihara H, Sakai T, Ohshiro K, Yamamoto T 2001 Phenotypic modulation of parietal epithelial cells of Bowman's capsule in culture. Cell Tissue Res 304:339-349

31. Mundel P, Reiser J, Kriz W 1997 Induction of differentiation in cultured rat and human podocytes. J Am Soc Nephrol 8:697-705

32. Durvasula RV, Shankland SJ 2006 Podocyte injury and targeting therapy: an update. Curr Opin Nephrol Hypertens 15:1-7

33. Marshall SM 2007 The podocyte: a potential therapeutic target in diabetic nephropathy? Curr Pharm Des 13:2713-2720
34. Barisoni L, Schnaper HW, Kopp JB 2007 A proposed taxonomy for the podocytopathies: a reassessment of the primary nephrotic diseases. Clin J Am Soc Nephrol 2:529-542

35. Lahdenkari AT, Lounatmaa K, Patrakka J, Holmberg C, Wartiovaara J, Kestila M, Koskimies O, Jalanko H 2004 Podocytes are firmly attached to glomerular basement membrane in kidneys with heavy proteinuria. J Am Soc Nephrol 15:2611-2618

36. Li JJ, Kwak SJ, Jung DS, Kim JJ, Yoo TH, Ryu DR, Han SH, Choi HY, Lee JE, Moon SJ, Kim DK, Han DS, Kang SW 2007 Podocyte biology in diabetic nephropathy. Kidney Int Suppl:S36-S42

37. Wiggins RC 2007 The spectrum of podocytopathies: a unifying view of glomerular diseases. Kidney Int 71:1205-1214

38. Vigneau C, Zheng F, Polgar K, Wilson PD, Striker G 2006 Stem cells and kidney injury. Curr Opin Nephrol Hypertens 15:238-244 